\title{
Iterative Methods for Solving the Fractional Form of Unsteady Axisymmetric Squeezing Fluid Flow with Slip and No-Slip Boundaries
}

\author{
A. A. Hemeda and E. E. Eladdad \\ Department of Mathematics, Faculty of Science, Tanta University, Tanta 31527, Egypt \\ Correspondence should be addressed to A. A. Hemeda; aahemeda@yahoo.com
}

Received 15 January 2016; Accepted 11 April 2016

Academic Editor: Giorgio Kaniadakis

Copyright (C) 2016 A. A. Hemeda and E. E. Eladdad. This is an open access article distributed under the Creative Commons Attribution License, which permits unrestricted use, distribution, and reproduction in any medium, provided the original work is properly cited.

\begin{abstract}
An unsteady axisymmetric flow of nonconducting, Newtonian fluid squeezed between two circular plates is proposed with slip and no-slip boundaries. Using similarity transformation, the system of nonlinear partial differential equations of motion is reduced to a single fourth-order nonlinear ordinary differential equation. By using the basic definitions of fractional calculus, we introduced the fractional order form of the fourth-order nonlinear ordinary differential equation. The resulting boundary value fractional problems are solved by the new iterative and Picard methods. Convergence of the considered methods is confirmed by obtaining absolute residual errors for approximate solutions for various Reynolds number. The comparisons of the solutions for various Reynolds number and various values of the fractional order confirm that the two methods are identical and therefore are suitable for solving this kind of problems. Finally, the effects of various Reynolds number on the solution are also studied graphically.
\end{abstract}

\section{Introduction}

The squeezing of an incompressible viscous fluid between two parallel plates is a fundamental type of flow that is frequently observed in many hydrodynamical tools and machines. Compression and injection molding, polymer processing, and modeling of lubrication systems are some practical examples of squeezing flows where their usage is found. The modeling and analysis of squeezing flow has been started in the nineteenth century and continues to receive significant attention due to its vast applications areas in biophysical and physical sciences. The first work in squeezing flows was laid down by Stefan [1] who developed an ad hoc asymptotic solution of Newtonian fluids. An explicit solution of the squeeze flow, considering inertial terms, has been established by Thorpe and Shaw [2]. However, P. S. Gupta and A. S. Gupta proved that the solution given in [2] fails to satisfy boundary conditions [3]. Verma [4] and Singh et al. [5] have established numerical solutions of the squeezing flow between parallel plates. Leider and Byron Bird performed theoretical analysis of power-law fluid between parallel disks [6]. Qayyum et al. present in [7] analysis of unsteady axisymmetric flow of nonconducting, Newtonian fluid squeezed between two circular plates with slip and no-slip boundaries using OHAM and in [8] the authors model and analyse the unsteady axisymmetric flow of nonconducting Newtonian fluid squeezed between two circular plates passing through porous medium channel with slip boundary condition using HPM. Furthermore, in [9] analytical solutions for squeeze flow with partial wall slip are introduced by Laun et al., while in [10] Ullah et al. present approximation of first-grade MHD squeezing fluid flow with slip boundary condition using DTM and OHAM. In [11] analytical solution of squeezing flow between two circular plates is presented by Rashidi et al. Islam et al. [12] studied Newtonian squeezing fluid flow in a porous medium channel, Siddiqui et al. [13] investigated the unsteady squeezing flow of viscous fluid with magnetic field, Marinca et al. [14] applied an optimal homotopy asymptotic method to the steady flow of a fourth-grade fluid past a porous plate, and Idrees et al. [15] applied the optimal homotopy asymptotic method to squeezing flow. For more details see $[16,17]$. 
A large number of perturbation methods, which can solve nonlinear boundary value problems analytically, are discussed in the literature. These methods, however, have a limitation of assuming small or large parameters. Recently, Daftardar-Gejji and Jafari have proposed a new technique, called new iterative method (NIM), for solving linear and nonlinear boundary value problems. The method minimize the limitations that are usually associated with perturbation methods and at the same time it takes full advantages of the traditional perturbation methods. This method has proven useful for solving a variety of nonlinear equations such as algebraic equations, integral equations, ordinary and partial differential equations of integer and fractional order, and systems of equations as well. The NIM is used by Hemeda in [18] for solving fractional partial differential equations and in [19] for solving the $n t h$-order integrodifferential equations, but in [20] he used the NIM for solving fractional physical differential equations. Also, this method is used by DaftardarGejji and Jafari in [21] for solving nonlinear fractional equations and by Bhalekar and Daftardar-Gejji in [22] it is used for solving partial differential equations while in [23] it is used for solving evolution equations. In the late of the nineteenth century, Emile Picard proposed a good method called Picard iteration method (or shortly) Picard method (PM), which is used widely by researches for solving linear and nonlinear boundary value problems. This method has not any of the difficulties presented in perturbation methods and takes full advantages of the traditional perturbation methods as well as NIM. The PM is used by Youssef and El-Arabawy in [24] for initial value problems and by Ibijola and Adegboyegun in [25] for solving nonlinear differential equations. Also, this method is used by Hemeda in [26] for solving the fractional gas dynamics and coupled Burgers' equations. In this method, the solution takes the form of a rapidly convergent series with easily computable components.

The importance of the study of the fractional forms of the differential equations is due to their wide appearing in many of the mathematical, physical, and chemical problems. So the aim of this work is to continue in this study by preparing and using the NIM and PM where there is not any of the above-mentioned difficulties in the perturbation methods for solving the fractional order form of an unsteady axisymmetric squeezing fluid flow between two circular plates with slip and no-slip boundaries. Also the effects of various of Reynolds number and fractional order on the solution are studied tabularly and graphically.

\section{Formulation of the Problem}

In this section, the unsteady axisymmetric squeezing flow of incompressible Newtonian fluid with density $\rho$, viscosity $\mu$, and kinematic viscosity $\nu$, squeezed between two circular plates having speed $E_{\omega}(t)$, is considered with a fractional form. It is assumed that, at any time $t$, the distance between the two circular plates is $2 h(t)$. Also it is assumed that $r$-axis is the central axis of the channel while $z$-axis is taken as normal to it. Plates move symmetrically with respect to the central axis $z=0$ while the flow is axisymmetric about $r=0$. The longitudinal and normal velocity components in radial and axial directions are $w_{r}(r, z, t)$ and $w_{z}(r, z, t)$, respectively. For more physical explanation and details, see $[7,8]$.

The equations of motion are

$$
\begin{aligned}
\frac{\partial w_{r}}{\partial r}+\frac{w_{r}}{r}+\frac{\partial w_{z}}{\partial z} & =0, \\
\frac{\partial p}{\partial r}+\rho\left(\frac{\partial w_{r}}{\partial t}-w_{z} \Omega\right) & =-\mu \frac{\partial \Omega}{\partial z}, \\
\frac{\partial p}{\partial z}+\rho\left(\frac{\partial w_{r}}{\partial t}+w_{r} \Omega\right) & =\frac{\mu}{r} \frac{\partial}{\partial r}(r \Omega),
\end{aligned}
$$

where $\Omega=\Omega(r, z, t)$ is the vorticity function and $p=$ $p(r, z, t)$ is the generalized pressure.

The boundary conditions on $w_{r}(r, z, t)$ and $w_{z}(r, z, t)$ are

$$
\begin{aligned}
& w_{r}(r, z, t)=0, \\
& w_{z}(r, z, t)=E_{\omega}(t) \\
& \text { at } z=h, \\
& \frac{\partial}{\partial z} w_{r}(r, z, t)=0, \\
& w_{z}(r, z, t)=0
\end{aligned}
$$

where $E_{\omega}(t)=d h / d t$ is the velocity of the plates. The boundary conditions in (4) are due to symmetry at $z=0$ and no-slip at the upper plate when $z=h$. If we introduce the dimensionless parameter

$$
\eta=\frac{z}{h(t)}
$$

(1), (2), and (3) transforms to

$$
\begin{gathered}
\frac{\partial w_{r}}{\partial r}+\frac{w_{r}}{r}+\frac{1}{h} \frac{\partial w_{z}}{\partial \eta}=0 \\
\frac{\partial p}{\partial r}+\rho\left(\frac{\partial w_{r}}{\partial t}-w_{z} \Omega\right)=-\frac{\mu}{h} \frac{\partial \Omega}{\partial \eta} \\
\frac{1}{h} \frac{\partial p}{\partial \eta}+\rho\left(\frac{\partial w_{r}}{\partial t}+w_{r} \Omega\right)=\frac{\mu}{r} \frac{\partial}{\partial r}(r \Omega) .
\end{gathered}
$$

The boundary conditions on $w_{r}$ and $w_{z}$ are

$$
\begin{aligned}
w_{r} & =0, \\
w_{z} & =E_{\omega}(t) \\
\quad \text { at } \eta=1, & \\
\frac{\partial}{\partial \eta} w_{r} & =0, \\
w_{z} & =0
\end{aligned}
$$


By eliminating the generalized pressure between (7) and (8), we obtain

$$
\rho\left[\frac{\partial \Omega}{\partial t}+w_{r} \frac{\partial \Omega}{\partial r}+\frac{w_{z}}{h} \frac{\partial \Omega}{\partial \eta}-\frac{w_{r}}{r} \Omega\right]=\mu\left[\nabla^{2} \Omega-\frac{\Omega}{r^{2}}\right],
$$

where $\nabla^{2}$ is the Laplacian operator.

Defining velocity components as [3]

$$
\begin{aligned}
& w_{r}=-\frac{r}{2 h(t)} E_{\omega}(t) u^{\prime}(\eta), \\
& w_{z}=E_{\omega}(t) u(\eta),
\end{aligned}
$$

we see that (6) is identically satisfied and (10) becomes

$$
\frac{d^{4} u}{d \eta^{4}}+R\left[(\eta-u) \frac{d^{3} u}{d \eta^{3}}+2 \frac{d^{2} u}{d \eta^{2}}\right]-Q \frac{d^{2} u}{d \eta^{2}}=0,
$$

where

$$
\begin{aligned}
R & =\frac{h E_{\omega}}{v}, \\
Q & =\frac{h^{2}}{v E_{\omega}} \frac{d E_{\omega}}{d t} .
\end{aligned}
$$

Here both $R$ and $Q$ are functions of $t$ but we consider $R$ and $Q$ as constants for similarity solution. Since $E_{\omega}=d h / d t$, integrate first equation of (13), and we get

$$
h(t)=(c t+d)^{1 / 2},
$$

where $c$ and $d$ are constants. The plates move away from each other symmetrically with respect to $\eta$ when $c>0$ and $d>0$. Also the plates approach each other and squeezing flow exists with similar velocity profiles when $c<0, d>0$, and $h(t)>0$. From (13) and (14), it follows that $Q=-R$. Then, (12) becomes

$$
\frac{d^{4} u}{d \eta^{4}}+R\left[(\eta-u) \frac{d^{3} u}{d \eta^{3}}+3 \frac{d^{2} u}{d \eta^{2}}\right]=0 .
$$

Using (9) and (11), we determine the boundary conditions in case of no-slip and slip at the upper plate as follows:

$$
\begin{gathered}
u(1)=1, \\
u^{\prime}(1)=0, \\
u(0)=0 \\
u^{\prime \prime}(0)=0
\end{gathered}
$$

(No-slip at the wall),

$$
\begin{aligned}
& u(1)=1, \\
& u^{\prime}(1)=\gamma u^{\prime \prime}(1), \\
& u(0)=0 \\
& u^{\prime \prime}(0)=0
\end{aligned}
$$

\section{Fractional Calculus}

In this section, we mention some basic definitions of fractional calculus which are used in the present work.

Definition 1. The Riemann-Liouville fractional integral operator of order $\alpha>0$, of a function $f(t) \in c_{\mu}$ and $\mu \geq-1$, is defined as [27]

$$
\begin{aligned}
I_{t}^{\alpha} f(t) & =\frac{1}{\Gamma(\alpha)} \int_{0}^{t}(t-\tau)^{\alpha-1} f(\tau) d \tau, \quad \alpha>0, t>0, \\
I_{t}^{0} f(t) & =f(t) .
\end{aligned}
$$

For the Riemann-Liouville fractional integral operator, $I_{t}^{\alpha}$, we obtain

$$
I_{t}^{\alpha} t^{\nu}=\frac{\Gamma(\nu+1) t^{\nu+\alpha}}{\Gamma(\nu+1+\alpha)} .
$$

Definition 2. The fractional derivative of $f(t)$ in the Caputo sense is defined as [28]

$$
\begin{aligned}
D_{t}^{\alpha} f(t) & =I^{m-\alpha} D_{t}^{m} f(t) \\
& =\frac{1}{\Gamma(m-\alpha)} \int_{0}^{t}(t-\tau)^{m-\alpha-1} f^{(m)}(\tau) d \tau, \\
& \quad \text { for } m-1<\alpha \leq m, t>0 .
\end{aligned}
$$
obtain

For the Caputo fractional derivative operator, $D_{t}^{\alpha}$, we

$$
D_{t}^{\alpha} t^{\nu}=\frac{\Gamma(\nu+1) t^{\nu-\alpha}}{\Gamma(\nu+1-\alpha)}
$$

For the Riemann-Liouville fractional integral and Caputo fractional derivative operator of order $\alpha$, we have the following relation:

$$
I_{t}^{\alpha} D_{t}^{\alpha} f(t)=f(t)-\sum_{k=0}^{m-1} f^{(k)}\left(0_{+}\right) \frac{t^{k}}{k !}
$$

Remark 3. According to the previous fractional calculus, (15) can be rewritten in the following fractional order form:

$$
\frac{d^{\alpha} u}{d \eta^{\alpha}}+R\left[(\eta-u) \frac{d^{3} u}{d \eta^{3}}+3 \frac{d^{2} u}{d \eta^{2}}\right]=0, \quad 3<\alpha \leq 4 .
$$

\section{Analysis of the Considered Methods}

In this section, we discuss the considered methods with preparing them for solving any fractional differential equation.

4.1. New Iterative Method (NIM). To illustrate the basic idea of this method, we consider the following general functional equation [18-23]:

$$
u(t)=f(t)+N(u(t)),
$$


where $N$ is a nonlinear operator from a Banach space $B \rightarrow B$ and $f(t)$ is a known function (element) of a Banach space $B$. We are looking for a solution $u(t)$ of (23) having the series form:

$$
u(t)=\sum_{i=0}^{\infty} u_{i}(t)
$$

The nonlinear operator $N$ can be decomposed as

$$
\begin{aligned}
N\left(\sum_{i=0}^{\infty} u_{i}\right)= & N\left(u_{0}\right) \\
& +\sum_{i=1}^{\infty}\left\{N\left(\sum_{j=0}^{i} u_{j}\right)-N\left(\sum_{j=0}^{i-1} u_{j}\right)\right\} .
\end{aligned}
$$

From (24) and (25), (23) is equivalent to

$$
\begin{aligned}
\sum_{i=0}^{\infty} u_{i}= & +N\left(u_{0}\right) \\
& +\sum_{i=1}^{\infty}\left\{N\left(\sum_{j=0}^{i} u_{j}\right)-N\left(\sum_{j=0}^{i-1} u_{j}\right)\right\} .
\end{aligned}
$$

The required solution $u(t)$ for (23) can be obtained recurrently from the recurrence relation:

$$
\begin{aligned}
u_{0} & =f \\
u_{1} & =N\left(u_{0}\right), \\
u_{r+1} & =N\left(\sum_{i=0}^{r} u_{i}\right)-N\left(\sum_{i=0}^{r-1} u_{i}\right), \quad r=1,2, \ldots
\end{aligned}
$$

Then,

$$
\begin{aligned}
& \sum_{i=1}^{r+1} u_{i}=N\left(\sum_{i=0}^{r} u_{i}\right), \quad r=0,1,2, \ldots, \\
& \sum_{i=0}^{\infty} u_{i}=f+N\left(\sum_{i=0}^{\infty} u_{i}\right) .
\end{aligned}
$$

The $r$-term approximate solution of (23) is given by

$$
u(t)=\sum_{i=0}^{r-1} u_{i}
$$

4.2. Solving General Fractional Differential Equation by NIM. To solve any fractional differential equation of arbitrary order $\alpha>0$, we consider the following general fractional differential equation of order $\alpha$ :

$$
\begin{aligned}
D_{t}^{\alpha} u(t)=g(t)+L(u(t))+ & K(u(t)), \\
& m-1<\alpha \leq m, m \in N,
\end{aligned}
$$

subject to the initial values

$$
\frac{d^{k}}{d t^{k}} u(0)=h_{k}, \quad k=0,1,2, \ldots, m-1,
$$

where $L$ is a linear operator, $K$ is a nonlinear operator, $g(t)$ is a nonhomogeneous term, and $D_{t}^{\alpha}$ is the fractional differential operator of order $\alpha>0$. In view of the fractional integral operators, the initial value fractional problem (30a) and (30b) is equivalent to the fractional integral equation:

$$
\begin{aligned}
u(t)= & \sum_{k=0}^{m-1} h_{k} \cdot \frac{t^{k}}{k !}+I_{t}^{\alpha}[g(t)] \\
& +I_{t}^{\alpha}[L(u(t))+K(u(t))]=f+N(u),
\end{aligned}
$$

where $f=\sum_{k=0}^{m-1} h_{k} \cdot\left(t^{k} / k !\right)+I_{t}^{\alpha}[g(t)], N(u)=I_{t}^{\alpha}[L(u(t))+$ $K(u(t))]$, and $I_{t}^{\alpha}$ is the inverse of $D_{t}^{\alpha}$. The required solution $u(t)$ for (31) and hence for (30a) and (30b) can be obtained recurrently from the recurrence relation (27).

4.3. Picard Method (PM). To illustrate the basic idea of this method, we consider the following general fractional differential equation of arbitrary order $\alpha>0$ [24-26]:

$$
\begin{aligned}
& D_{t}^{\alpha} u(t)=F\left(t, u^{(k)}(t)\right), \\
& \qquad m-1<\alpha \leq m, m \in N, \\
& \frac{d^{k}}{d t^{k}} u(0)=h_{k}, \quad k=0,1,2, \ldots, m-1,
\end{aligned}
$$

where $D_{t}^{\alpha}$ is the fractional differential operator of order $\alpha>$ 0 . In view of the fractional integral operators, the initial value fractional problem (32a) and (32b) is equivalent to the fractional integral equation:

$$
u(t)=\sum_{k=0}^{m-1} h_{k} \cdot \frac{t^{k}}{k !}+I_{t}^{\alpha}\left[F\left(t, u_{t}^{(k)}(t)\right)\right]=f+N(u)
$$

where $f=\sum_{k=0}^{m-1} h_{k} \cdot\left(t^{k} / k !\right), N(u)=I_{t}^{\alpha}\left[F\left(t, u_{t}^{(k)}(t)\right)\right]$, and $I_{t}^{\alpha}$ is the inverse of $D_{t}^{\alpha}$. The required solution $u(t)$ for (33) which is also the solution for (32a) and (32b) can be obtained as the limit of a sequence of functions $u_{r+1}(t)$ generated by the recurrence relation:

$$
\begin{aligned}
u_{0} & =f, \\
u_{r+1} & =u_{0}+N\left(u_{r}\right), \quad r=0,1,2, \ldots,
\end{aligned}
$$

where $u(t)=\lim _{r \rightarrow \infty} u_{r}(t)$.

\section{Applications}

In this section, we illustrate the application of the two considered methods to solve the nonlinear fractional order ordinary differential equation (22) subject to the boundary conditions (16a) and (16b). 
5.1. NIM. Using (22), (16a), and (16b), the initial value fractional order problem

$$
\begin{aligned}
\frac{d^{\alpha} u}{d \eta^{\alpha}}+R\left[(\eta-u) \frac{d^{3} u}{d \eta^{3}}+3 \frac{d^{2} u}{d \eta^{2}}\right] & =0, \quad 3<\alpha \leq 4 \\
u(0) & =0 \\
u^{\prime}(0) & =a \\
u^{\prime \prime}(0) & =0 \\
u^{\prime \prime \prime}(0) & =b
\end{aligned}
$$

according to (31), is equivalent to the fractional integral equation:

$$
u(\eta)=a \eta+\frac{b \eta^{3}}{6}-I_{\eta}^{\alpha}\left[R\left((\eta-u) \frac{d^{3} u}{d \eta^{3}}+3 \frac{d^{2} u}{d \eta^{2}}\right)\right]
$$

where $a$ and $b$ are constants. Let $N(u)=-I_{\eta}^{\alpha}[R((\eta-$ $\left.\left.u)\left(d^{3} u / d \eta^{3}\right)+3\left(d^{2} u / d \eta^{2}\right)\right)\right]$. Therefore, according to (27), we can obtain the following first few components of the new iterative solution for (35)

$$
\begin{aligned}
u_{0}(\eta) & =a \eta+\frac{b \eta^{3}}{6}, \\
u_{1}(\eta) & =\frac{a b R \eta^{1+\alpha}}{\Gamma(2+\alpha)}-\frac{4 b R \eta^{1+\alpha}}{\Gamma(2+\alpha)}+\frac{b^{2} R \eta^{3+\alpha}}{\Gamma(4+\alpha)} \\
u_{2}(\eta) & =\frac{4 b R^{2}(\alpha-1) \eta^{-1+2 \alpha}}{\Gamma(2 \alpha)}-\frac{5 a b R^{2}(\alpha-1) \eta^{-1+2 \alpha}}{\Gamma(2 \alpha)} \\
+ & \frac{a^{2} b R^{2} \eta^{-1+2 \alpha}}{\Gamma(2 \alpha)}-\frac{2 b^{2} R^{2} \Gamma(2+\alpha) \eta^{1+2 \alpha}}{3 \Gamma(-1+\alpha) \Gamma(2+2 \alpha)}+\cdots
\end{aligned}
$$

and so on. In the same manner, the rest of components can be obtained. The 4-term solution for (35) in series form is given by

$$
\begin{aligned}
u(\eta)= & \sum_{i=0}^{3} u_{i} \\
= & a \eta+\frac{b \eta^{3}}{6}+\frac{a b R \eta^{1+\alpha}}{\Gamma(2+\alpha)}-\frac{4 b R \eta^{1+\alpha}}{\Gamma(2+\alpha)}+\frac{b^{2} R \eta^{3+\alpha}}{\Gamma(4+\alpha)} \\
& +\frac{4 b R^{2}(\alpha-1) \eta^{-1+2 \alpha}}{\Gamma(2 \alpha)} \\
& -\frac{5 a b R^{2}(\alpha-1) \eta^{-1+2 \alpha}}{\Gamma(2 \alpha)}+\frac{a^{2} b R^{2} \eta^{-1+2 \alpha}}{\Gamma(2 \alpha)}
\end{aligned}
$$

In the special case, $\alpha=4$, (38) becomes

$$
\begin{aligned}
u(\eta)= & a \eta+\frac{b \eta^{3}}{6}-\frac{b R \eta^{5}}{30}+\frac{a b R \eta^{5}}{120}+\frac{b^{2} R \eta^{7}}{5040} \\
& +\frac{b R^{2} \eta^{7}}{210}-\frac{a b R^{2} \eta^{7}}{280}+\cdots
\end{aligned}
$$

Using the boundary conditions in (16a) with the initial conditions in (35), the unknowns $a$ and $b$ for fixed values of $R$ in (39) can be easily determined. In case of no-slip boundary, then $a=1.5$ and $b=-3.0$. For $R=0.5$, the 4-term solution, obtained by the NIM in (39), is therefore

$$
\begin{aligned}
u(\eta)= & 1.5 \eta-0.5 \eta^{3}+0.03125 \eta^{5}+0.000334821 \eta^{7} \\
& -0.000171673 \eta^{9}+1.02166 \times 10^{-6} \eta^{11} \\
& +1.36077 \times 10^{-6} \eta^{13}-2.6881 \times 10^{-8} \eta^{15} \\
& -2.68729 \times 10^{-9} \eta^{17}+1.23186 \times 10^{-10} \eta^{19} \\
& +3.42474 \times 10^{-12} \eta^{21}-1.79838 \times 10^{-13} \eta^{23} \\
& -4.69921 \times 10^{-15} \eta^{25}+7.12005 \times 10^{-17} \eta^{27} \\
& +2.18728 \times 10^{-18} \eta^{29}+1.18008 \times 10^{-20} \eta^{31}
\end{aligned}
$$

Similarly, using the boundary conditions in (16b) with the initial conditions in (35), in case of slip boundary, then $a=$ 0.75 and $b=1.5$. For $R=0.5$, the 4 -term solution becomes

$$
\begin{aligned}
u(\eta)= & 0.75 \eta+0.25 \eta^{3}-0.0203125 \eta^{5} \\
& +0.00113002 \eta^{7}-8.87674 \times 10^{-5} \eta^{9} \\
& +6.75394 \times 10^{-6} \eta^{11}-4.50029 \times 10^{-7} \eta^{13} \\
& +2.4254 \times 10^{-8} \eta^{15}-1.00801 \times 10^{-9} \eta^{17} \\
& +3.48556 \times 10^{-11} \eta^{19}-1.05106 \times 10^{-12} \eta^{21} \\
& +2.53779 \times 10^{-14} \eta^{23}-4.22141 \times 10^{-16} \eta^{25} \\
& +4.23058 \times 10^{-18} \eta^{27}-2.22146 \times 10^{-20} \eta^{29} \\
& +4.6097 \times 10^{-23} \eta^{31} .
\end{aligned}
$$

5.2. PM. Also the initial value fractional problem (35), according to (33) and (34), is equivalent to the fractional integral equation:

$$
\begin{array}{r}
u_{r+1}(\eta)=u_{0}-I_{\eta}^{\alpha}\left[R\left(\left(\eta-u_{r}\right) \frac{d^{3} u_{r}}{d \eta^{3}}+3 \frac{d^{2} u_{r}}{d \eta^{2}}\right)\right] \\
r=0,1,2, \ldots
\end{array}
$$


Therefore, according to (34), we can obtain the following first few components of Picard solution for (35):

$$
\begin{aligned}
& u_{0}(\eta)=a \eta+\frac{b \eta^{3}}{6}, \\
& u_{1}(\eta)=a \eta+\frac{b \eta^{3}}{6}+\frac{a b R \eta^{1+\alpha}}{\Gamma(2+\alpha)}-\frac{4 b R \eta^{1+\alpha}}{\Gamma(2+\alpha)} \\
& +\frac{b^{2} R \eta^{3+\alpha}}{\Gamma(4+\alpha)}, \\
& u_{2}(\eta)=a \eta+\frac{b \eta^{3}}{6}+\frac{a b R \eta^{1+\alpha}}{\Gamma(2+\alpha)}-\frac{4 b R \eta^{1+\alpha}}{\Gamma(2+\alpha)} \\
& +\frac{b^{2} R \eta^{3+\alpha}}{\Gamma(4+\alpha)}+\frac{4 b R^{2}(\alpha-1) \eta^{-1+2 \alpha}}{\Gamma(2 \alpha)} \\
& -\frac{5 a b R^{2}(\alpha-1) \eta^{-1+2 \alpha}}{\Gamma(2 \alpha)}+\frac{a^{2} b R^{2} \eta^{-1+2 \alpha}}{\Gamma(2 \alpha)} \\
& -\frac{2 b^{2} R^{2} \Gamma(2+\alpha) \eta^{1+2 \alpha}}{3 \Gamma(-1+\alpha) \Gamma(2+2 \alpha)}+\cdots
\end{aligned}
$$

and so on. In the same manner the rest of components can be obtained. The 4-order term solution for (35), in series form, is given by

$$
\begin{aligned}
u_{3}(\eta)= & a \eta+\frac{b \eta^{3}}{6}+\frac{a b R \eta^{1+\alpha}}{\Gamma(2+\alpha)}-\frac{4 b R \eta^{1+\alpha}}{\Gamma(2+\alpha)} \\
& +\frac{b^{2} R \eta^{3+\alpha}}{\Gamma(4+\alpha)}+\frac{4 b R^{2}(\alpha-1) \eta^{-1+2 \alpha}}{\Gamma(2 \alpha)} \\
& -\frac{5 a b R^{2}(\alpha-1) \eta^{-1+2 \alpha}}{\Gamma(2 \alpha)}+\frac{a^{2} b R^{2} \eta^{-1+2 \alpha}}{\Gamma(2 \alpha)} \\
& -\cdots
\end{aligned}
$$

In the special case, $\alpha=4$, (44) becomes

$$
\begin{aligned}
u_{3}(\eta)= & a \eta+\frac{b \eta^{3}}{6}-\frac{b R \eta^{5}}{30}+\frac{a b R \eta^{5}}{120}+\frac{b^{2} R \eta^{7}}{5040} \\
& +\frac{b R^{2} \eta^{7}}{210}-\frac{a b R^{2} \eta^{7}}{280}+\cdots
\end{aligned}
$$

Also, in case of no-slip boundary where $R=0.5$, the 4 order term solution is

$$
\begin{aligned}
u(\eta)= & 1.5 \eta-0.5 \eta^{3}+0.03125 \eta^{5}+0.000334821 \eta^{7} \\
& -0.000171673 \eta^{9}+1.02166 \times 10^{-6} \eta^{11}
\end{aligned}
$$

$$
\begin{aligned}
& +1.36077 \times 10^{-6} \eta^{13}-2.6881 \times 10^{-8} \eta^{15} \\
& -2.68729 \times 10^{-9} \eta^{17}+1.23186 \times 10^{-10} \eta^{19} \\
& +3.42474 \times 10^{-12} \eta^{21}-1.79838 \times 10^{-13} \eta^{23} \\
& -4.69921 \times 10^{-15} \eta^{25}+7.12005 \times 10^{-17} \eta^{27} \\
& +2.18728 \times 10^{-18} \eta^{29}+1.18008 \times 10^{-20} \eta^{31}
\end{aligned}
$$

and, in case of slip boundary, the 4-order term solution becomes

$$
\begin{aligned}
u(\eta)= & 0.75 \eta+0.25 \eta^{3}-0.0203125 \eta^{5} \\
& +0.00113002 \eta^{7}-8.87674 \times 10^{-5} \eta^{9} \\
& +6.75394 \times 10^{-6} \eta^{11}-4.50029 \times 10^{-7} \eta^{13} \\
& +2.4254 \times 10^{-8} \eta^{15}-1.00801 \times 10^{-9} \eta^{17} \\
& +3.48556 \times 10^{-11} \eta^{19}-1.05106 \times 10^{-12} \eta^{21} \\
& +2.53779 \times 10^{-14} \eta^{23}-4.22141 \times 10^{-16} \eta^{25} \\
& +4.23058 \times 10^{-18} \eta^{27}-2.22146 \times 10^{-20} \eta^{29} \\
& +4.6097 \times 10^{-23} \eta^{31} .
\end{aligned}
$$

From the previous results for (35), obtained by the two considered methods, it is clear that the approximate solution for (35) obtained by PM in (44), (45), (46), and (47) is the same approximate solution as obtained by NIM in (38), (39), (40), and (41). Therefore, the two methods are identical in solving this problem and hence the two methods are suitable for solving this kind of problems.

The residual error of the problem is

$$
\begin{aligned}
\operatorname{Re}(\eta) & =\text { Residual Error } \\
& =\frac{d^{\alpha} \widehat{u}}{d \eta^{\alpha}}+R\left[(\eta-\widehat{u}) \frac{d^{3} \widehat{u}}{d \eta^{3}}+3 \frac{d^{2} \widehat{u}}{d \eta^{2}}\right],
\end{aligned}
$$

where $\widehat{u}$ is the 4-term approximate solution in (38) or (44) for (35).

If $\operatorname{Re}=0$, then $\widehat{u}$ will be the exact solution. However, this usually does not occur in nonlinear problems.

It is clear from the obtained results that the aboveconsidered methods minimize the limitations of the ordinary perturbation methods. In the same time, these methods take full advantages of the traditional perturbation methods. Therefore, these methods are powerful methods for solving the nonlinear fractional order differential equations.

\section{Numerical Results and Discussion}

In this work, an unsteady axisymmetric flow of nonconducting, incompressible Newtonian fluid squeezed between two circular plates is considered. The resulting nonlinear 
TABLE 1: Solutions for different values of $\eta$ and $\alpha$ at $R=0.1$, in case of no-slip boundary.

\begin{tabular}{lcccccc}
\hline$\eta$ & $\alpha=3.7$ & $\alpha=3.8$ & $\alpha=3.9$ & $\alpha=4.0$ & \multicolumn{2}{c}{$\alpha=4.1$} \\
\hline 0.0 & 0.0 & 0.0 & 0.0 & 0.0 & 0.0 & 0.0 \\
0.1 & 0.149500 & 0.149500 & 0.149500 & 0.149500 & 0.149500 & $5.63438^{-15}$ \\
0.2 & 0.296005 & 0.296004 & 0.296003 & 0.296002 & 0.296001 & $7.21923^{-13}$ \\
0.3 & 0.436536 & 0.436527 & 0.436520 & 0.436515 & 0.436511 & $1.14405^{-11}$ \\
0.4 & 0.568140 & 0.568108 & 0.568083 & 0.568064 & 0.568049 & $5.97444^{-11}$ \\
0.5 & 0.687900 & 0.687816 & 0.687750 & 0.687697 & 0.687654 & $2.94232^{-11}$ \\
0.6 & 0.792945 & 0.792761 & 0.792611 & 0.792490 & 0.792392 & $2.46997^{-9}$ \\
0.7 & 0.880455 & 0.880099 & 0.879805 & 0.879563 & 0.879364 & $2.03243^{-8}$ \\
0.8 & 0.947673 & 0.947045 & 0.946519 & 0.946080 & 0.945714 & $1.09632^{-7}$ \\
0.9 & 0.991913 & 0.990880 & 0.990004 & 0.989263 & 0.988637 & $4.67357^{-7}$ \\
1.0 & 1.010560 & 1.008960 & 1.00758 & 1.006400 & 1.005390 & $1.69343^{-6}$ \\
\hline
\end{tabular}

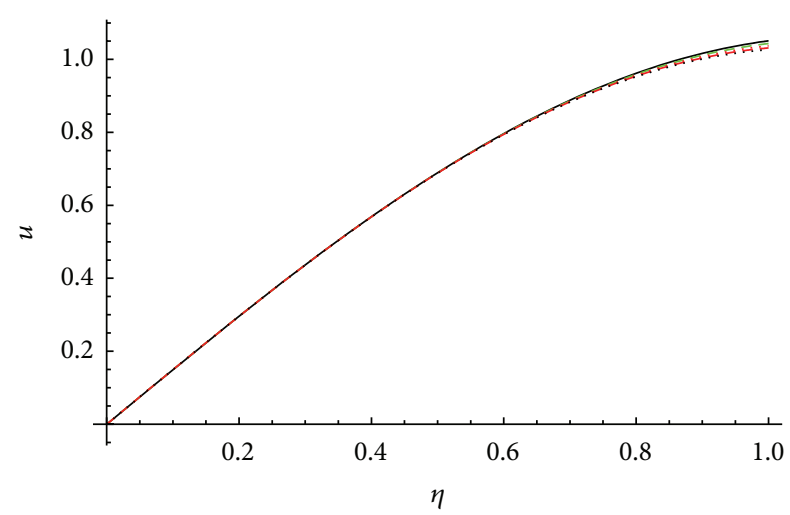

Figure 1: Solution for $\alpha=3.7,3.8,3.9,4.0,4.1$ at $R=0.5$ in case of no-slip boundary.

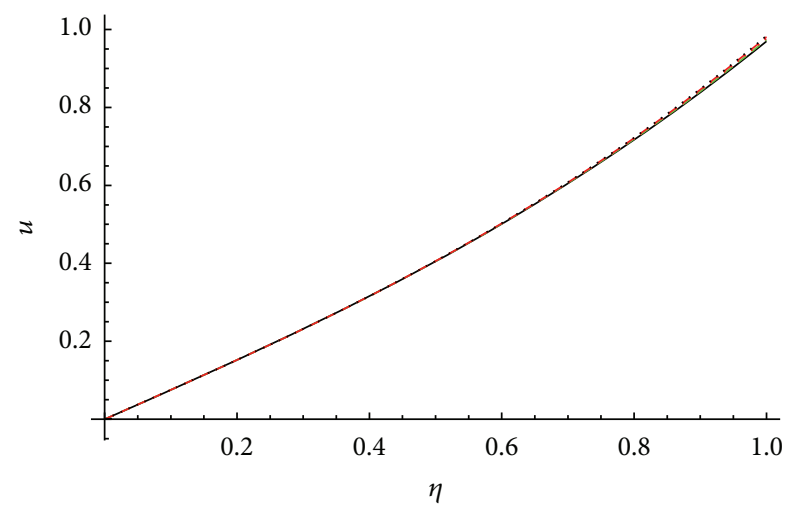

FIGURE 2: Solution for $\alpha=3.7,3.8,3.9,4.0,4.1$ at $R=0.5, \gamma=1$ in case of slip boundary.

fractional order boundary value problem is solved analytically in cases of no-slip and slip boundaries using NIM and PM.

Tables 1, 2, and 3 present the solutions in case of no-slip boundary for different values of the fractional order $\alpha$ along with absolute residual errors $|\operatorname{Re}|$ at $\alpha=4$ for fixed value of the Reynolds number $R$ in each table, while Tables 4, 5, and 6 are in case of slip boundary and slip parameter $\gamma=1$. The results in the six tables indicate that, as $R \rightarrow 0$ and $\alpha \rightarrow 4$,

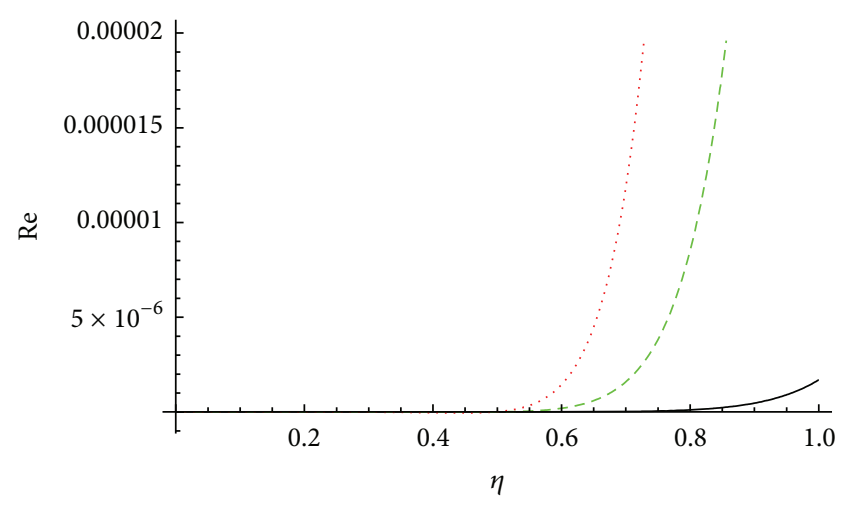

FIGURE 3: Residual for $R=0.1$ (continuous curve), 0.3 (dashed curve), and 0.5 (dotted curve) at $\alpha=4.0$ in case of no-slip boundary.

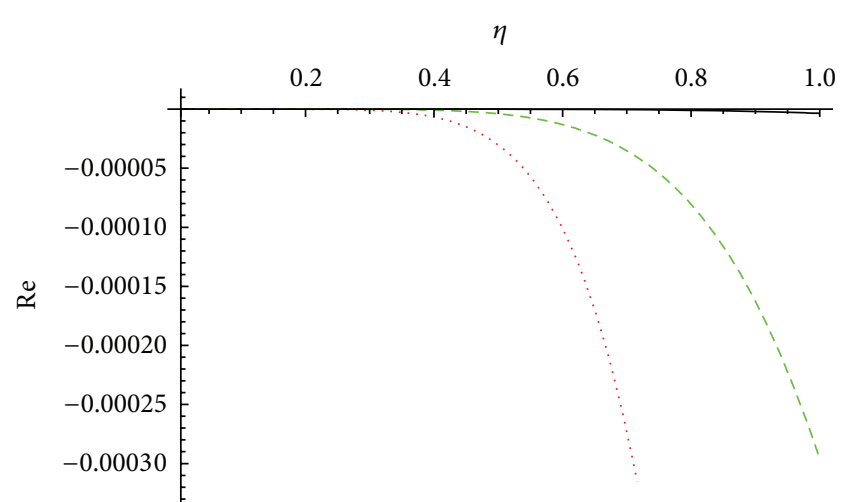

Figure 4: Residual for $R=0.1$ (continuous curve), 0.3 (dashed curve), and 0.5 (dotted curve) at $\alpha=4.0, \gamma=1$ in case of slip boundary.

$|\operatorname{Re}| \rightarrow 0$. Therefore, the approximate solutions converge to the exact solution for the considered problem.

Figures 1 and 2 indicate the approximate solutions for different values of $\alpha=3.7,3.8,3.9,4.0,4.1$ at $R=0.5$ in cases of no-slip and slip boundaries, respectively, with $\gamma=$ 1. Figures 3 and 4 show the residual errors Re at $\alpha=4$ for different values of $R=0.1,0.3,0.5$ in cases of no-slip and slip boundaries, respectively, with $\gamma=1$. In addition 
TABLE 2: Solutions for different values of $\eta$ and $\alpha$ at $R=0.3$, in case of no-slip boundary.

\begin{tabular}{|c|c|c|c|c|c|c|}
\hline$\eta$ & $\alpha=3.7$ & $\alpha=3.8$ & $\alpha=3.9$ & $\alpha=4.0$ & $\alpha=4.1$ & $|\operatorname{Re}|_{\alpha=4}$ \\
\hline 0.0 & 0.0 & 0.0 & 0.0 & 0.0 & 0.0 & 0.0 \\
\hline 0.1 & 0.149501 & 0.149500 & 0.149500 & 0.149500 & 0.149500 & $4.55608^{-13}$ \\
\hline 0.2 & 0.296016 & 0.296012 & 0.296008 & 0.296006 & 0.296004 & $5.84710^{-11}$ \\
\hline 0.3 & 0.436608 & 0.436581 & 0.436561 & 0.436546 & 0.436534 & $9.26826^{-10}$ \\
\hline 0.4 & 0.568418 & 0.568324 & 0.568250 & 0.568193 & 0.568148 & $4.85819^{-9}$ \\
\hline 0.5 & 0.688694 & 0.688445 & 0.688247 & 0.688088 & 0.687963 & $1.90307^{-9}$ \\
\hline 0.6 & 0.794814 & 0.794270 & 0.793827 & 0.793467 & 0.793175 & $1.93785^{-7}$ \\
\hline 0.7 & 0.884309 & 0.883263 & 0.882394 & 0.881676 & 0.881085 & $1.59180^{-6}$ \\
\hline 0.8 & 0.954885 & 0.953051 & 0.951504 & 0.950206 & 0.949120 & $8.52601^{-6}$ \\
\hline 0.9 & 1.004440 & 1.001450 & 0.998887 & 0.996708 & 0.994859 & $3.59984^{-5}$ \\
\hline 1.0 & 1.03108 & 1.026470 & 1.022470 & 1.019020 & 1.01606 & $1.28930^{-4}$ \\
\hline
\end{tabular}

TABLE 3: Solutions for different values of $\eta$ and $\alpha$ at $R=0.5$, in case of no-slip boundary.

\begin{tabular}{|c|c|c|c|c|c|c|}
\hline$\eta$ & $\alpha=3.7$ & $\alpha=3.8$ & $\alpha=3.9$ & $\alpha=4.0$ & $\alpha=4.1$ & $|\operatorname{Re}|_{\alpha=4}$ \\
\hline 0.0 & 0.0 & 0.0 & 0.0 & 0.0 & 0.0 & 0.0 \\
\hline 0.1 & 0.149501 & 0.149501 & 0.149500 & 0.149500 & 0.149500 & $3.51580^{-12}$ \\
\hline 0.2 & 0.296027 & 0.296019 & 0.296014 & 0.296010 & 0.296007 & $4.51141^{-10}$ \\
\hline 0.3 & 0.436680 & 0.436635 & 0.436602 & 0.436576 & 0.436557 & $7.15258^{-9}$ \\
\hline 0.4 & 0.568695 & 0.568538 & 0.568416 & 0.568321 & 0.568247 & $3.76300^{-8}$ \\
\hline 0.5 & 0.689480 & 0.689070 & 0.688741 & 0.688479 & 0.688270 & $1.10362^{-8}$ \\
\hline 0.6 & 0.796656 & 0.795763 & 0.795033 & 0.794438 & 0.793954 & $1.44776^{-6}$ \\
\hline 0.7 & 0.888089 & 0.886381 & 0.884956 & 0.883773 & 0.882795 & $1.18733^{-5}$ \\
\hline 0.8 & 0.961917 & 0.958942 & 0.956417 & 0.954287 & 0.952498 & $6.31480^{-5}$ \\
\hline 0.9 & 1.016570 & 1.011760 & 1.007600 & 1.004050 & 1.001010 & $2.64053^{-4}$ \\
\hline 1.0 & 1.050790 & 1.043440 & 1.037000 & 1.031420 & 1.026570 & $9.34686^{-4}$ \\
\hline
\end{tabular}

TABLE 4: Solutions for different values of $\eta$ and $\alpha$ at $R=0.1$ and $\gamma=1$ in case of slip boundary.

\begin{tabular}{lcccccc}
\hline$\eta$ & $\alpha=3.7$ & $\alpha=3.8$ & $\alpha=3.9$ & $\alpha=4.0$ & $\alpha=4.1$ & 0.0 \\
0.0 & 0.0 & 0.0 & 0.0 & 0.0 & 0.0 \\
0.1 & 0.075250 & 0.075250 & 0.075250 & 0.075250 & 0.075250 & 0.151999 \\
0.2 & 0.151997 & 0.151997 & 0.151998 & 0.151999 & 0.231743 & $9.13051^{-11}$ \\
0.3 & 0.231727 & 0.231732 & 0.231737 & 0.231740 & $1.50939^{-9}$ \\
0.4 & 0.315910 & 0.315930 & 0.315946 & 0.315959 & 0.315968 & $1.07916^{-8}$ \\
0.5 & 0.405994 & 0.406047 & 0.406090 & 0.406124 & 0.406151 & 0.503749 \\
0.6 & 0.503398 & 0.503514 & 0.503609 & 0.503686 & 0.610200 & $1.60795^{-7}$ \\
0.7 & 0.609512 & 0.609735 & 0.609921 & 0.610074 & 0.726916 & $4.31515^{-7}$ \\
0.8 & 0.725690 & 0.726081 & 0.726410 & 0.726685 & 0.855278 & $9.85311^{-7}$ \\
0.9 & 0.853250 & 0.853886 & 0.854429 & 0.854889 & 0.996637 & $1.97750^{-6}$ \\
1.0 & 0.993468 & 0.994447 & 0.995291 & 0.996016 & $3.56318^{-6}$ \\
\hline
\end{tabular}

to the above figures, the effect of Reynolds number $R$ on velocity profiles in case of no-slip boundary is shown in Figure 5. In these profiles, we varied $R$ as $R=0.1,0.3,0.5$ and observed that the normal velocity is increased with increasing
$R$ (Figure 5(a)). It is also noted that the normal velocity monotonically increases from $\eta=0$ to $\eta=1$ for fixed value of $R$ at a given time. Figure 5(b) describes the effect of $R$ on the longitudinal velocity in case of no-slip boundary. It is clear 
TABLE 5: Solutions for different values of $\eta$ and $\alpha$ at $R=0.3$ and $\gamma=1$ in case of slip boundary.

\begin{tabular}{|c|c|c|c|c|c|c|}
\hline$\eta$ & $\alpha=3.7$ & $\alpha=3.8$ & $\alpha=3.9$ & $\alpha=4.0$ & $\alpha=4.1$ & $|\operatorname{Re}|_{\alpha=4}$ \\
\hline 0.0 & 0.0 & 0.0 & 0.0 & 0.0 & 0.0 & 0.0 \\
\hline 0.1 & 0.075250 & 0.075250 & 0.075250 & 0.075250 & 0.075250 & $5.89262^{-11}$ \\
\hline 0.2 & 0.151990 & 0.151992 & 0.151995 & 0.151996 & 0.151997 & $7.39595^{-9}$ \\
\hline 0.3 & 0.231680 & 0.231697 & 0.231711 & 0.231720 & 0.231728 & $1.22281^{-7}$ \\
\hline 0.4 & 0.315732 & 0.315792 & 0.315839 & 0.315876 & 0.315905 & $8.74585^{-7}$ \\
\hline 0.5 & 0.405489 & 0.405646 & 0.405772 & 0.405873 & 0.405953 & $3.92681^{-6}$ \\
\hline 0.6 & 0.502219 & 0.502559 & 0.502838 & 0.503065 & 0.503250 & $1.30608^{-5}$ \\
\hline 0.7 & 0.607104 & 0.607750 & 0.608290 & 0.608738 & 0.609110 & $3.51392^{-5}$ \\
\hline 0.8 & 0.721230 & 0.722348 & 0.723298 & 0.724099 & 0.724775 & $8.05617^{-5}$ \\
\hline 0.9 & 0.845586 & 0.847384 & 0.848935 & 0.850263 & 0.851400 & $1.62693^{-4}$ \\
\hline 1.0 & 0.981059 & 0.983786 & 0.986174 & 0.988246 & 0.990047 & $2.95870^{-4}$ \\
\hline
\end{tabular}

TABLE 6: Solutions for different values of $\eta$ and $\alpha$ at $R=0.5$ and $\gamma=1$ in case of slip boundary.

\begin{tabular}{|c|c|c|c|c|c|c|}
\hline$\eta$ & $\alpha=3.7$ & $\alpha=3.8$ & $\alpha=3.9$ & $\alpha=4.0$ & $\alpha=4.1$ & $|\operatorname{Re}|_{\alpha=4}$ \\
\hline 0.0 & 0.0 & 0.0 & 0.0 & 0.0 & 0.0 & 0.0 \\
\hline 0.1 & 0.075250 & 0.075250 & 0.075250 & 0.075250 & 0.075250 & $4.54678^{-10}$ \\
\hline 0.2 & 0.151983 & 0.151987 & 0.151991 & 0.151994 & 0.151995 & $5.70694^{-8}$ \\
\hline 0.3 & 0.231634 & 0.231663 & 0.231684 & 0.231701 & 0.231713 & $9.43682^{-7}$ \\
\hline 0.4 & 0.315556 & 0.315655 & 0.315733 & 0.315794 & 0.315841 & $6.75191^{-6}$ \\
\hline 0.5 & 0.404995 & 0.405251 & 0.405458 & 0.405624 & 0.405756 & $3.03391^{-6}$ \\
\hline 0.6 & 0.501075 & 0.501625 & 0.502079 & 0.502451 & 0.502756 & $1.01056^{-4}$ \\
\hline 0.7 & 0.604788 & 0.605823 & 0.606696 & 0.607426 & 0.608035 & $2.72554^{-4}$ \\
\hline 0.8 & 0.716984 & 0.718754 & 0.720276 & 0.721570 & 0.722671 & $6.27313^{-4}$ \\
\hline 0.9 & 0.838373 & 0.841182 & 0.843641 & 0.845764 & 0.847608 & $1.27436^{-3}$ \\
\hline 1.0 & 0.969523 & 0.973721 & 0.977461 & 0.980735 & 0.983639 & $2.33762^{-3}$ \\
\hline
\end{tabular}

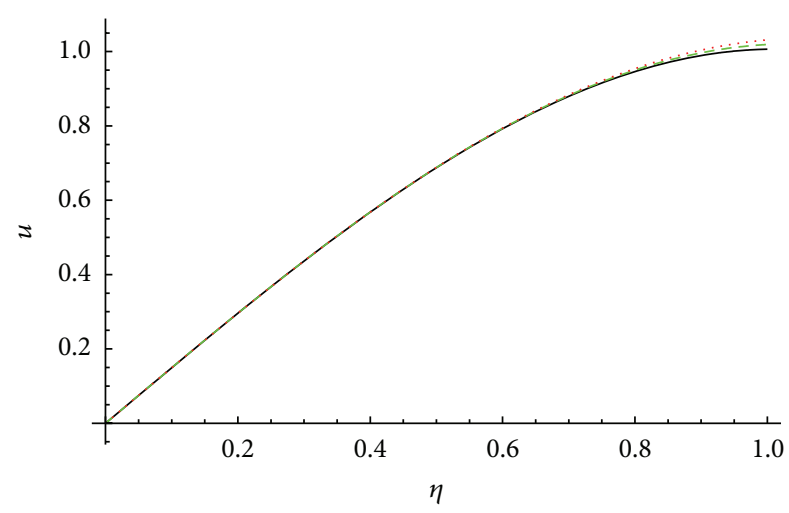

(a)

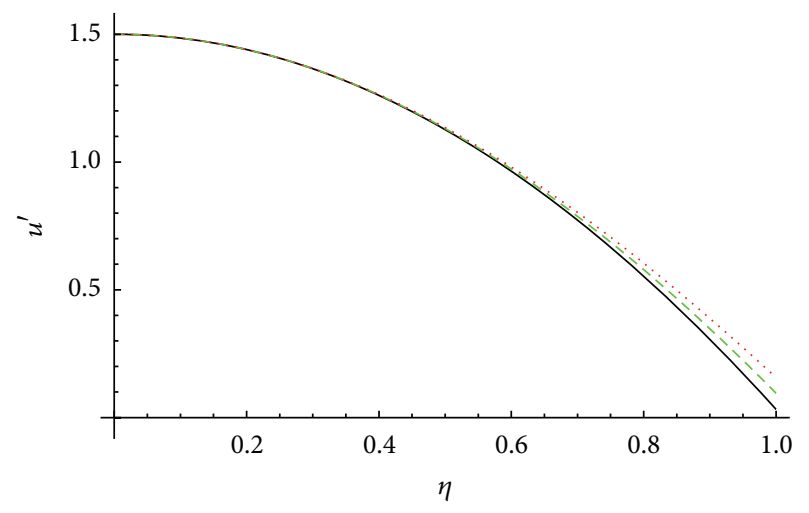

(b)

Figure 5: (a) Velocity profiles (normal velocity) for $R=0.1$ (continuous curve), 0.3 (dashed curve), and 0.5 (dotted curve) at $\alpha=4.0$ in case of no-slip boundary. (b) Velocity profiles (longitudinal velocity) for $R=0.1$ (continuous curve), 0.3 (dashed curve), and 0.5 (dotted curve) at $\alpha=4.0$ in case of no-slip boundary. 


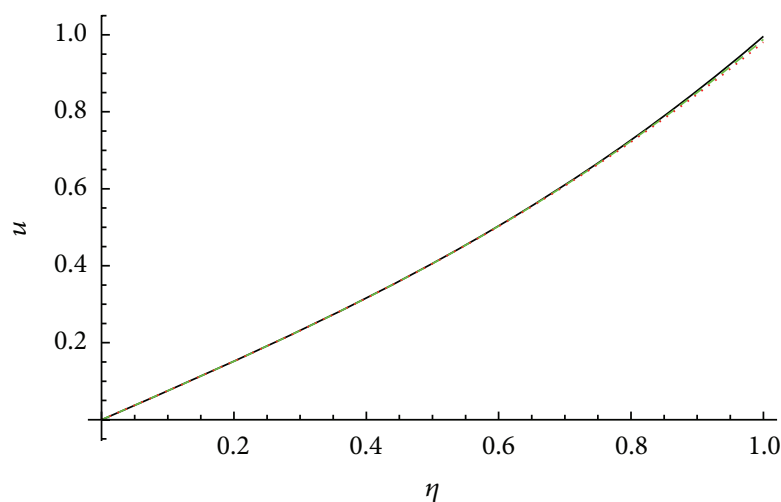

(a)

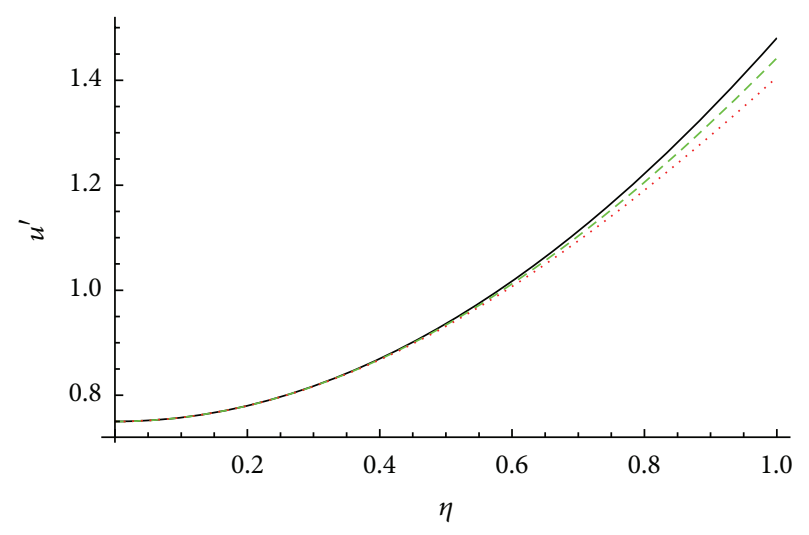

(b)

Figure 6: (a) Velocity profiles (normal velocity) for $R=0.1$ (continuous curve), 0.3 (dashed curve), and 0.5 (dotted curve) at $\alpha=4.0, \gamma=1$ in case of slip boundary. (b) Velocity profiles (longitudinal velocity) for $R=0.1$ (continuous curve), 0.3 (dashed curve), and 0.5 (dotted curve) at $\alpha=4.0, \gamma=1$ in case of slip boundary.

that this component of velocity decreases near the wall but increases near the central axis of the channel.

The effect of Reynolds number $R$ on velocity profiles in case of slip boundary is depicted in Figure 6. In these profiles, we varied $R$ as $R=0.1,0.3,0.5$ for fixed slip parameter $\gamma=1$. It is noted that the normal velocity decreases as $R$ increases (Figure 6(a)). It is also noted that the longitudinal velocity decreases near the central axis of the channel but increases near the walls when $R$ increases (Figure 6(b)).

\section{Conclusion}

In this work, an analytical solution for an unsteady axisymmetric squeezing flow of incompressible Newtonian fluid between two circular plates in fractional form is obtained using the NIM and PM in cases of no-slip and slip boundaries. Analysis of the residual errors confirms that the NIM and $\mathrm{PM}$ are identical and efficient schemes. Convergence of the considered methods is confirmed by absolute residual errors for different values of the Reynolds number $R$. Therefore, we concluded that the considered methods can be effectively used in various fields of science and engineering as they give better results in terms of accuracy.

\section{Competing Interests}

The authors declare that there are no competing interests regarding the publication of this paper.

\section{References}

[1] M. J. Stefan, "Versuch über die scheinbare adhäsion," Sitzungsberichte/Österreichische Akademie der Wissenschaften in Wien Mathematisch-Naturwissenschaftliche Klasse, vol. 69, pp. 713$721,1874$.

[2] J. F. Thorpe and W. A. Shaw, Developments in Theoretical and Applied Mechanics, Pergamon Press, Oxford, UK, 1976.

[3] P. S. Gupta and A. S. Gupta, "Squeezing flow between parallel plates," Wear, vol. 45, no. 2, pp. 177-185, 1977.
[4] R. L. Verma, "A numerical solution for squeezing flow between parallel channels," Wear, vol. 72, no. 1, pp. 89-95, 1981.

[5] P. Singh, V. Radhakrishnan, and K. A. Narayan, "Squeezing flow between parallel plates," Ingenieur-Archiv, vol. 60, no. 4, pp. 274281, 1990.

[6] P. J. Leider and R. Byron Bird, "Squeezing flow between parallel disks. I. Theoretical analysis," Industrial and Engineering Chemistry Fundamentals, vol. 13, no. 4, pp. 336-341, 1974.

[7] M. Qayyum, H. Khan, M. T. Rahim, and I. Ullah, "Analysis of unsteady axisymmetric squeezing fluid flow with slip and no-slip boundaries using OHAM," Mathematical Problems in Engineering, vol. 2015, Article ID 860857, 11 pages, 2015.

[8] M. Qayyum, H. Khan, M. T. Rahim, and I. Ullah, "Modeling and analysis of unsteady axisymmetric squeezing fluid flow through porous medium channel with slip boundary," PLoS ONE, vol. 10, no. 3, article e0117368, 2015.

[9] H. M. Laun, M. Rady, and O. Hassager, "Analytical solutions for squeeze flow with partial wall slip," Journal of Non-Newtonian Fluid Mechanics, vol. 81, no. 1-2, pp. 1-15, 1999.

[10] I. Ullah, H. Khan, and M. T. Rahim, "Approximation of first grade MHD squeezing fluid flow with slip boundary condition using DTM and OHAM," Mathematical Problems in Engineering, vol. 2013, Article ID 816262, 9 pages, 2013.

[11] M. M. Rashidi, A. M. Siddiqui, and M. T. Rastegari, "Analytical solution of squeezing flow between two circular plates," International Journal for Computational Methods in Engineering Science and Mechanics, vol. 13, no. 5, pp. 342-349, 2012.

[12] S. Islam, H. Khan, I. A. Shah, and G. Zaman, "An axisymmetric squeezing fluid flow between the two infinite parallel plates in a porous medium channel," Mathematical Problems in Engineering, vol. 2011, Article ID 349803, 10 pages, 2011.

[13] A. M. Siddiqui, S. Irum, and A. R. Ansari, "Unsteady squeezing flow of a viscous MHD fluid between parallel plates, a solution using the homotopy perturbation method," Mathematical Modelling and Analysis, vol. 13, no. 4, pp. 565-576, 2008.

[14] V. Marinca, N. Herişanu, C. Bota, and B. Marinca, "An optimal homotopy asymptotic method applied to the steady flow of a fourth-grade fluid past a porous plate," Applied Mathematics Letters, vol. 22, no. 2, pp. 245-251, 2009.

[15] M. Idrees, S. Islam, S. Haq, and S. Islam, "Application of the optimal homotopy asymptotic method to squeezing flow," 
Computers \& Mathematics with Applications, vol. 59, no. 12, pp. 3858-3866, 2010.

[16] R. J. Grimm, "Squeezing flows of Newtonian liquid films an analysis including fluid inertia," Applied Scientific Research, vol. 32, no. 2, pp. 149-166, 1976.

[17] H. Khan, S. Islam, J. Ali, and I. Ali Shah, "Comparison of different analytic solutions to axisymmetric squeezing fluid flow between two infinite parallel plates with slip boundary conditions," Abstract and Applied Analysis, vol. 2012, Article ID 835268, 18 pages, 2012.

[18] A. A. Hemeda, "Solution of fractional partial differential equations in fluid mechanics by extension of some iterative method," Abstract and Applied Analysis, vol. 2013, Article ID 717540, 9 pages, 2013.

[19] A. A. Hemeda, "New iterative method: application to nth-order integro-differential equations," INFORMATION, Japan, vol. 16, no. 6, pp. 3841-3852, 2011.

[20] A. A. Hemeda, "New iterative method: an application for solving fractional physical differential equations," Abstract and Applied Analysis, vol. 2013, Article ID 617010, 9 pages, 2013.

[21] V. Daftardar-Gejji and H. Jafari, "An iterative method for solving nonlinear functional equations," Journal of Mathematical Analysis and Applications, vol. 316, no. 2, pp. 753-763, 2006.

[22] S. Bhalekar and V. Daftardar-Gejji, "New iterative method: application to partial differential equations," Applied Mathematics and Computation, vol. 203, no. 2, pp. 778-783, 2008.

[23] S. Bhalekar and V. Daftardar-Gejji, "Solving evolution equations using a new iterative method," Numerical Methods for Partial Differential Equations, vol. 26, no. 4, pp. 906-916, 2010.

[24] I. K. Youssef and H. A. El-Arabawy, "Picard iteration algorithm combined with Gauss-Seidel technique for initial value problems," Applied Mathematics and Computation, vol. 190, no. 1, pp. 345-355, 2007.

[25] E. A. Ibijola and B. J. Adegboyegun, "A comparison of Adomian's decomposition method and Picard iteration method in solving nonlinear differential equations," Global Journal of Science Frontier Research F: Mathematics and Decision Sciences, vol. 12, Version 1.0, no. 7, 2012.

[26] A. A. Hemeda, "Iterative methods for solving fractional gas dynamics and coupled Burgers' equations," International Journal of Mathematical Modelling and Numerical Optimisation, vol. 6, no. 4, pp. 282-298, 2015.

[27] I. Podlubny, Fractional Differential Equations, vol. 198 of Mathematics in Science and Engineering, Academic Press, San Diego, Calif, USA, 1999.

[28] M. Caputo, Elasticita E Dissipazione, Zani-Chelli, Bologna, Italy, 1969. 


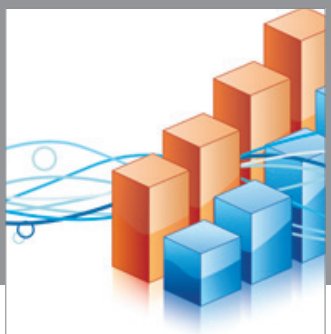

Advances in

Operations Research

vatem alat4

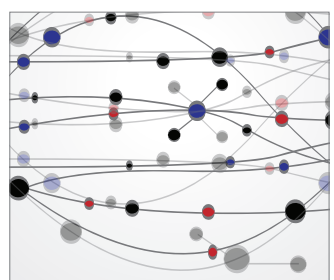

\section{The Scientific} World Journal
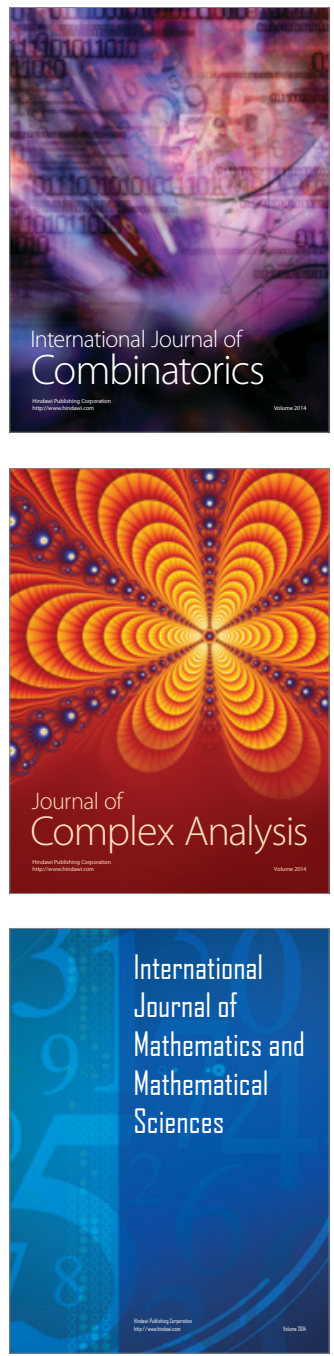
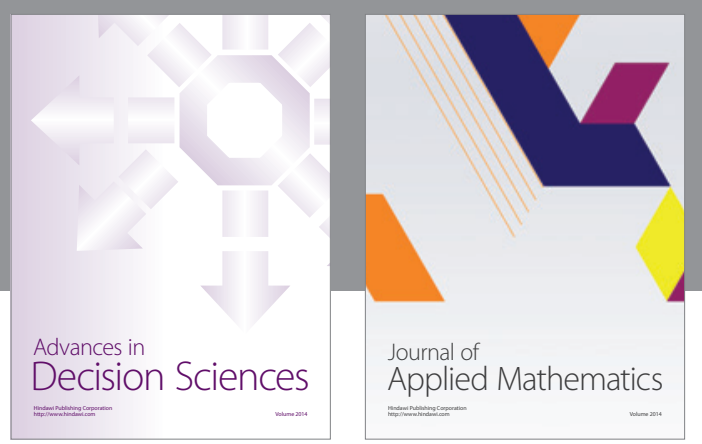

Algebra

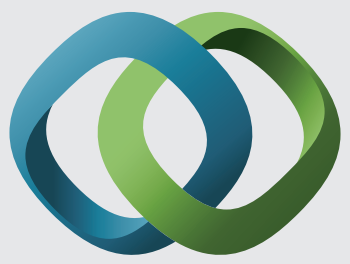

\section{Hindawi}

Submit your manuscripts at

http://www.hindawi.com
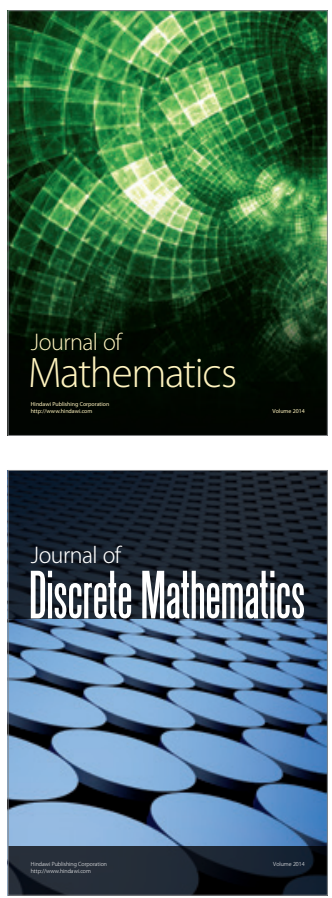

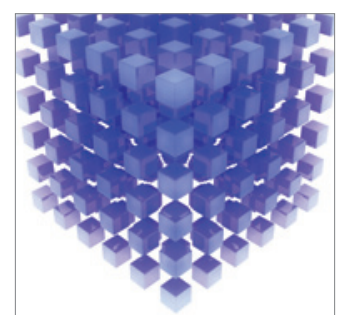

Mathematical Problems in Engineering
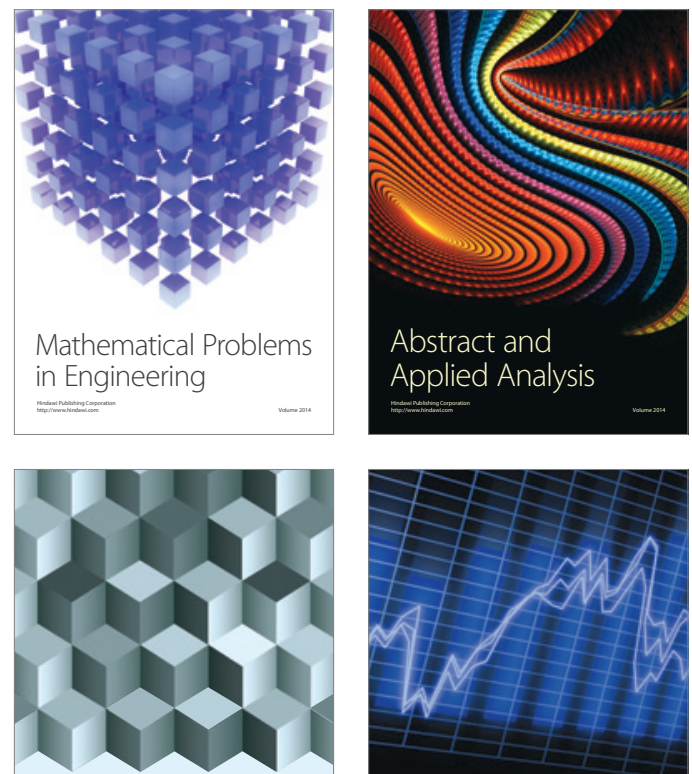

Journal of

Function Spaces

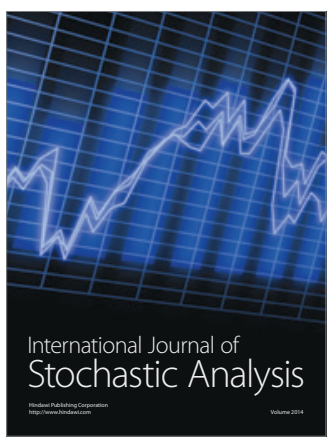

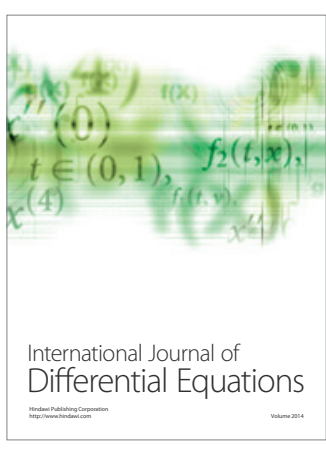
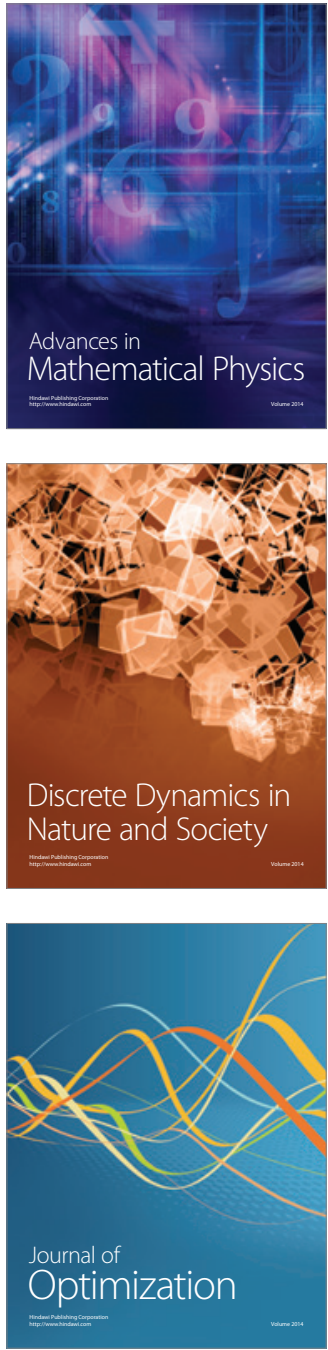